\title{
EDITORIAL
}

\section{EL ESTUDIO EMECAM SOBRE LOS EFECTOS DE LA CONTAMINACIÓN ATMOSFÉRICA}

\section{Jordi Sunyer}

Unitat de Recerca Respiratòria i Ambiental. IMIM. Barcelona.

Las evidencias de que los niveles actuales de contaminación del aire de nuestras ciudades pueden perjudicar la salud son cada vez mayores (ver la excelente revisión que presentan Tenías y col.). Al esfuerzo de la epidemiología se han sumado en los últimos años las evidencias toxicológicas y de estudios de laboratorio en voluntarios que sugieren, cada vez con mas coherencia científica, que las partículas provocan un daño a nivel pulmonar que tiene repercusiones sistémicas $^{1}$, como el aumento de la coagulabilidad ${ }^{2}$ o las alteraciones del ritmo cardíaco ${ }^{3}$. La habilidad de las partículas finas (menores a $2.5 \mu \mathrm{m}$ ) de penetrar por los bronquios hasta llegar a los alvéolos, su persistencia en el ambiente del interior de las casas, la presencia de partículas en el aire de todas las ciudades (a diferencia de los gases que son más específicos scgún cl tipo de ciudad), y la demostración, en estudios con voluntarios con lavado broncoalveolar de una gran reacción inflamatoria ${ }^{4}$, sugieren que ellas son las mayores responsables de dichos efectos. A pesar del largo camino recorrido, primero por la epidemiología y ahora por la toxicología, quedan todavía muchas preguntas abiertas. Si las partículas se asocian con efectos en la salud ¿cuales son las partículas responsables, las de pequeño tamaño o finas, y/o las aún menores o ultrafinas? ¿Es el número de partículas, su densidad o su cualidad química lo que explica el efecto? Y, finalmente ¿qué papel juegan los contaminantes en fase gas, son promotores de las partículas o también tienen un papel independiente?

En este contexto ¿cual es la aportación del estudio EMECAM? EMECAM es un es- tudio epidemiológico multicéntrico, sobre los efectos agudos de la contaminación urbana, que abarca las mayores ciudades españolas, así como aquellas que tuvieron un pasado reciente de alta contaminación de origen industrial, y una muestra de ciudades de mediano tamaño. EMECAM ha hecho el esfuerzo de reunir a un gran número de investigadores de distintas disciplinas, de diversos centros universitarios y de la administración sanitaria. El resultado ha sido el intercambio de conocimientos y la realización de un protocolo estándar (elaborado a partir de los conocimientos adquiridos en el estudio internacional $A P H E A$ ) por todos los centros, con un riguroso control en la recogida y análisis de los datos. La suma de esfuerzos ha permitido conocer, por primera vez, cual es la situación de un problema de salud pública, como la contaminación atmosférica en nuestro entorno, del que hasta la fecha sólo había información de escasos centros. El análisis conjunto de todas las ciudades revelará una información de interés para la salud pública. Los resultados presentados aquí, son específicos para cada ciudad, pero una visión conjunta permite observar una asociación significativa en varias ciudades, entre la contaminación atmosférica por partículas y la mortalidad, principalmente cardiovascular y respiratoria (tabla 1). ¿Significa ello que la contaminación causa un aumento de la mortalidad en las ciudades españolas? ¿Por qué no encontramos un efecto en todas las ciudades?

El estudio $E M E C A M$ utiliza un diseño propio de los estudios ambientales para investigar efectos agudos, como es el diseño 
Tabla 1

Riesgo relativo de morir por cada incremento en $10 \mu \mathrm{g} / \mathrm{m}^{3}$ en las partículas (promedio de 24 horas del mismo día o 3 días anteriores), según los artículos publicados a continuación

\begin{tabular}{|c|c|c|c|c|c|}
\hline \multirow{2}{*}{ Ciudad } & \multirow{2}{*}{ Indicador $\dagger$} & \multicolumn{3}{|c|}{$R R$ según causa mortalidad } & \multirow{2}{*}{ Otros contaminantes } \\
\hline & & Total & Cardio-vascular & Respiratoria & \\
\hline Barcelona 1991-1995 & - & - & - & - & $\mathrm{NO}_{2}, \mathrm{O}_{3}$ \\
\hline Bilbao 1992-1996 & $\begin{array}{c}\text { Humos N. } \\
\text { TSP }\end{array}$ & $\begin{array}{c}1.009 \\
1.008^{*}\end{array}$ & $1.012 *$ & $\begin{array}{l}1.030 \\
1.017\end{array}$ & $\mathrm{NO}_{2}$ \\
\hline Castelló 1991-1995 & Humos $\mathrm{N}$. & 1.019 & $1.032 *$ & - & - \\
\hline Cartagena 1992-1996 & TSP & 0.999 & 1.001 & 1.002 & -- \\
\hline Huelva 1993-1996 & PMI0 & 1.025 & - & 1.076 & - \\
\hline Gijón 1993-1996 & TSP & 1.006 & $\rightarrow$ & 0.978 & - \\
\hline Madrid 1992-1995 & PM10 & - & $1.009 *$ & 1.008 & $\mathrm{SO}_{2}, \mathrm{CO}$ \\
\hline Oviedo 1993-1996 & $\begin{array}{c}\text { Humos } \mathrm{N} . \\
\text { TSP }\end{array}$ & $0 . \overline{994}$ & $\begin{array}{l}1.015 \\
1.014\end{array}$ & $\begin{array}{l}1.026 \\
1.018\end{array}$ & -- \\
\hline Pamplona 1991-1995 & Humos N. & 1.030 & - & 1.134 & - \\
\hline Sevilla 1992-1996 & PM10 & -- & -- & 0.975 & $\mathrm{NO}_{2}$ \\
\hline Valencia 1994-1996 & Humos N. & $1.013^{*}$ & -- & - & $\mathrm{NO}_{2}, \mathrm{CO}$ \\
\hline Vigo 1991-1994 & Humos N. & 1.004 & - & - & $\mathrm{SO}_{2}$ \\
\hline Vitoria 1990-1994 & Humos N. & 1.006 & -- & -- & $\cdots$ \\
\hline Zaragoza 1991-995 & Humos N. & - & - & $1.028 *$ & $\mathrm{SO}_{2}$ \\
\hline
\end{tabular}

† Las partículas son medidas como lumns negros (Hunos N.), Partículas suspendidas totales (TSP), o partículas de tamaño menor de $10 \mu m$ (PM10).

$\ddagger$ Contaminantes gaseosos que muestran una asociación estadísticamente significativa $(p<0.05)$.

$* \mathrm{p}<0.05$

- No se presentan datos en los artículos publicados en esta revista para retrasos menores de 4 días.

de series temporales. Sáez y col. y Pérez-Hoyos y col. revisan la complejidad que comporta su aplicación de forma repetida en todos los centros. Estos estudios investigan los cambios que ocurren entre días sucesivos, de modo que la unidad de análisis es el día. La ventaja de este diseño es que incluye a todos los habitantes de una ciudad, sin ningún límite en la selección. El mayor inconveniente del diseño es la gran dificultad en modclizar correctamente las series. Cómo ajustar por las variaciones en la tendencia o la estación, cómo controlar por las variables meteorológicas, o cuáles son los retrasos en el efecto a medir, son decisiones que pueden afectar la bondad de los resultados. En un estudio ambiental, con variaciones en el ambiente de las ciudades, escoger la misma estrategia para modelizar tendencia, estación, clima y el retraso en cada ciudad, puede conllevar discrepancias en la estimación de los efectos. Se conoce que el número de muertes diarias influye en la adecuación del modelo matemático, y que los métodos paramétricos pueden no ser los adecuados cuando el número es pequeño, requiriéndose métodos de suavizado no-paramétricos difíciles de homogeneizar. Pero, así y todo, siempre queda la incógnita de si se ha ajustado correctamente por las variables meteorológicas. Es por ello, que los estudios de series temporales aportan una visión de los 
efectos con datos disponibles a partir de los registros de mortalidad y de contaminación, pero no son suficientes para concluir que las asociaciones observadas son causales. Además, se debe modelizar el retraso entre la exposición aguda y la aparición del efecto, que en $E M E C A M$ se decide de manera empírica a partir de los resultados de cada serie de datos. Sin embargo, retrasos de más de 3 días interfieren en la modelización de la autocorrelación propia de las series temporales. Finalmente, un problema común en los estudios epidemiológicos ambientales es la validez de la medida de la exposición, no sólo por su componente comunitario en lugar de personal, sino por la selección del indicador de contaminación. En estos momentos sc crec que el indicador adecuado para medir partículas es el PM2.5 (partículas de tamaño menor de $2.5 \mu \mathrm{m}$ ). Sin embargo, los errores en la medida de la exposición son no diferenciales y conllevan una subestimación de la asociación.

A la espera del análisis conjunto de todos los datos de EMECAM, la presente publicación sugiere que, en varias de las ciudades investigadas (Bilbao, Castelló, Madrid, Valencia, Vigo, Zaragoza para partículas y además Barcelona, Sevilla y Vigo para gases), la contaminación actual se asocia con la mortalidad (tabla 1). Sin embargo, la asociación encontrada es muy débil. Los riesgos relativos son menores de 1.1. Ello puede ser debido a la infraestimación producida por los errores en la medida de la exposición, pero señala una gran incógnita sobre el valor causal de los resultados. Sin embargo, dado que toda la población de una ciudad está expuesta a la contaminación atmosférica, el incremento de un $2 \%$ por cada 10 $\mu \mathrm{g} / \mathrm{m}^{3}$ puede tener un impacto considerable sobre la salud pública, cuya magnitud depende del tamaño de las ciudades. Ello es lo que ha llevado a algunos gobiernos a actuar a partir de información similar a la aportada por $E M E C A M$, sin esperar la respuesta a las preguntas todavía no resueltas. Estas actuaciones se dirigen a reducir las fuentes de contaminación urbana, como la reducción del espacio para el tránsito rodado, o la reducción del valor que indica atmósfera contaminada. Los investigadores de $E M E C A M$ han aportado una nueva pieza de información, presentada de forma preliminar en este número y que, tras el análisis conjunto de los datos, merecerá una respuesta sobre si es necesario actuar en nuestro entorno urbano.

\section{BIBLIOGRAFÍA}

1. Li XY, Gilmour PS, Donaldson K, MacNee W. Free radical activity and pro-inflammatory effects of particulate air pollution (PM10) in vivo and in vitro. Thorax 1996; 1216-22.

2. Peters A, Döring A, Wichman HE, Koenig W. Increased plasma viscosity during an air pollution episode: a link to mortality. Lancet 197; 349:1582-7.

3. Gold DR, Litonjua A, Scwartz J, Verrier M, Miltsein R, Larson A, et al. Cardiovascular vulnerability to particulate pollution. Am J Respir Crit Care Med 1998; 155:A268.

4. Salvi SS, Blomberg A, Rudell B, Kelly FJ, Sandstrom T, Holgate ST, Frew AJ. Acute inflammatory changes in the airways of healthy human subjects following short term exposure to diesel exhaust. Am J Resp Crit Care Med 1997:155: A425. 